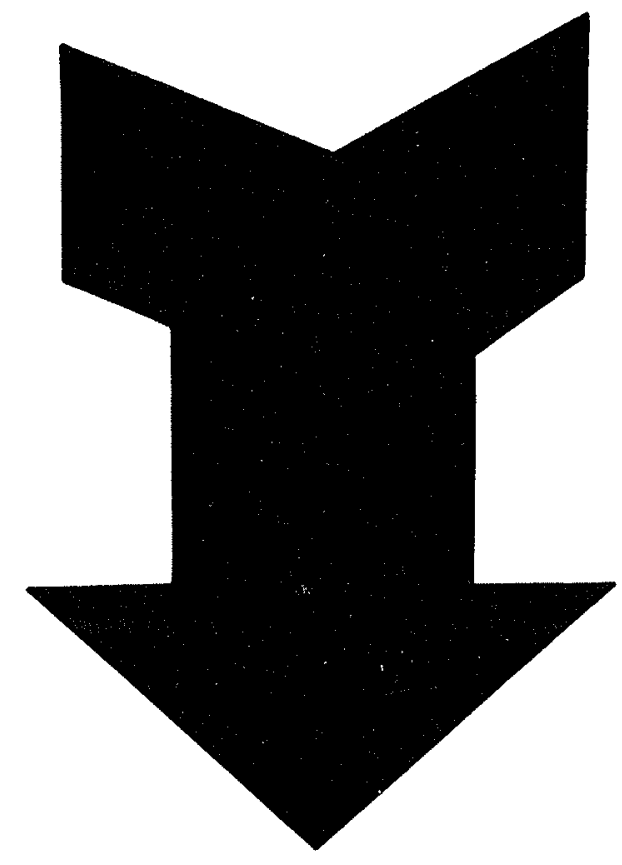

\title{
1967, UNE ANNÉE \\ TRÈS PARTICULIÈRE POUR L'ÉVOLUTION DE L'ÉTANG DE BERRE
}

PAR A. KIENER * ET R. LONGUEMARE * *
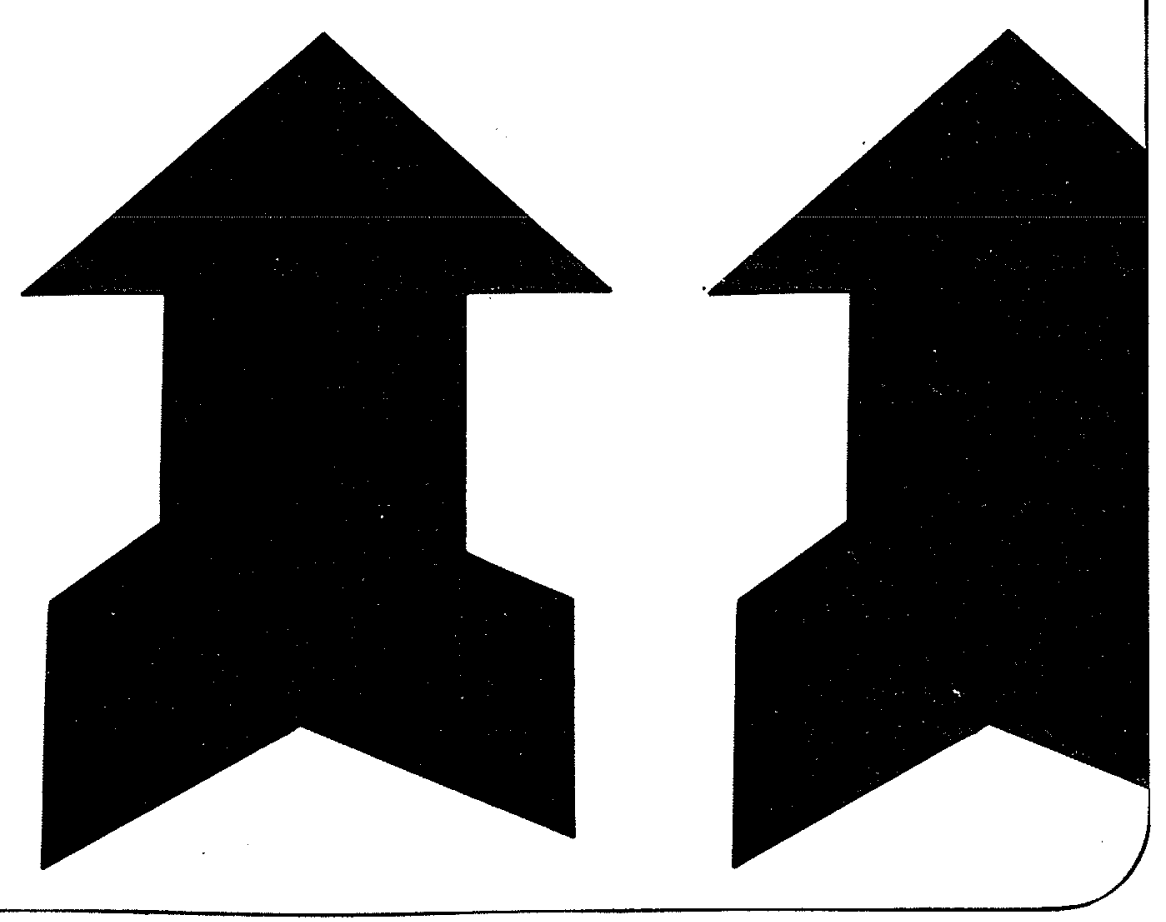

1967 :

Une année exceptionnelle au point de vue météorologique

Dans notre précédente communication (1967), rappelée en bibliographie, un premier bilan avait été dressé à la fin de l'année 1966 en vue de préciser certains aspects de l'évolution physique et biologique de l'étang de Berre provoquée par la dérivation des eaux de la Durance.

Une seconde année s'est écoulée et nous avons pensé qu'il serait intéressant de revoir ce bilan. Les conclusions tirées de notre première note n'étaient que partielles, l'exploitation des usines de la BasseDurance ayant seulement porté sur une durée de neuf mois. On pouvait espérer qu'en 1967, avec une exploitation normale de l'usine de Saint-Chamas, l'étang de Berre se serait fortement rapproché des conditions d'équilibre final prévu.

Or les prévisions en la matière ont été complètement bouleversées par une météorologie très particulière : 1967 fut une année à précipitations excep-

C.N.R.S. Laboratoire de Biologie Générale-Ecologie, Faculté des Sciences Saint-Charles, Marseille.

** E.D.F. Région d'Equipement Hydraulique Alpes-Sud. tionnellement faibles et un ensoleillement intense qui provoqua une forte évaporation. La Provence a subi l'année dernière un climat semi-aride comparable à celui d'Amman en Jordanie ou de Sfax en Tunisie, avec moins de $300 \mathrm{~mm}$ de pluie. Le tableau 1 donne les principales caractéristiques météorologiques de l'année, en rappelant certaines moyennes auxquelles il est intéressant de les comparer. La pluviométrie totalisant 221,7 $\mathrm{mm}$ en 1967 (fig. 1 a) représente le record de sécheresse sur cent cinquante ans de mesures durant lesquels deux années seulement présentent des précipitations inférieures à $300 \mathrm{~mm}$ : 1854 avec $298,5 \mathrm{~mm}$ el 1921 avec $282,5 \mathrm{~mm}$.

Du point de vue de la température, 1967 a été un peu plus chaud que 1966 et la moyenne annuelle a marqué presque un degré de plus que le chiffre moyen obtenu sur trente ans de mesures, ce qui est appréciable (fig. $1 \mathrm{~b}$ ). On comprendra donc aisément que l'évaporation a été particulièrement élevée (fig. $1 c$ c). Malgré une moindre intensité générale des vents par rapport aux autres années (le vent est un facteur desséchant bien connu et combien apprécié dans les salines !), l'évaporation a alteint $1860 \mathrm{~mm}$, entraînant un déficit évaporation-pluies de près de $1640 \mathrm{~mm}$. Le Service météo, en raison des dangers de gel pour ses appareils, ne procède plus depuis quelques années à la mesure de l'évaporation de novembre à mars, mais nous avons pu estimer l'évaporation pendant cette période grâce à d'anciens chiffres relevés en années chaudes et sèches. 


\begin{tabular}{|c|c|c|c|c|c|c|c|c|c|}
\hline \multirow[b]{3}{*}{ Mors } & \multicolumn{9}{|c|}{$\begin{array}{r}\text { Tableau } 1 \\
\text { Statistiques météorologiques }\left(^{*}\right) \text { pour la région de l'étang de Berre }\end{array}$} \\
\hline & \multicolumn{3}{|c|}{ ANNBE 1967} & \multicolumn{3}{|c|}{ ANx́̃e 1966} & \multicolumn{3}{|c|}{ Moyexaes longues priniones } \\
\hline & $\begin{array}{l}\text { Pluies } \\
(\mathrm{mm}) \\
\end{array}$ & $\begin{array}{l}\text { Evapo- } \\
\text { ration } \\
\text { (mm) }\end{array}$ & $\begin{array}{l}\mathrm{T} \\
\left({ }^{\circ} \mathrm{C}\right)\end{array}$ & $\begin{array}{l}\text { Pluies } \\
(\mathrm{mm})\end{array}$ & $\begin{array}{l}\text { Evapo- } \\
\text { ration } \\
(\mathrm{mm})\end{array}$ & $\begin{array}{c}\mathrm{T} \\
\left({ }^{\circ} \mathrm{C}\right) \\
\end{array}$ & $\begin{array}{c}\text { Pluies } \\
\text { 1946-1965 } \\
\text { (mm) }\end{array}$ & $\begin{array}{c}\text { Evapo- } \\
\text { ration } \\
1951-1961 \\
(\mathrm{~mm}) \\
\end{array}$ & $\begin{array}{c}\mathrm{T} \\
1931-1960 \\
\left({ }^{\circ} \mathrm{C}\right)\end{array}$ \\
\hline Janvier. . . . ....... & 15,9 & $100(* *)$ & 6,3 & 44,1 & $70\left({ }^{*}\right)$ & 6,2 & 38 & $70\left({ }^{*}\right)$ & 5,5 \\
\hline Février. . . ....... & 9,6 & $120(* *)$ & 8,5 & 98,6 & $80(* *)$ & 10,6 & 38 & $80(* *)$ & 6,6 \\
\hline Mars. . . ......... & 9,1 & $130(* *)$ & 11,8 & 2,7 & $100\left({ }^{* *}\right)$ & 9,9 & 44 & $100\left({ }^{* *}\right)$ & 10 \\
\hline Avril. . . . ....... & 18,8 & 173,1 & 13,5 & 39,4 & 117,4 & 14,4 & 38 & 147 & 13 \\
\hline Mai. . . . . . . . & 7,2 & 198,7 & 17,4 & 25,1 & 161,9 & 17,6 & 42 & 176 & 16,8 \\
\hline Juin. . . . . . . . . . & 22,5 & 213,4 & 20 & 21,9 & 220,3 & 21,7 & 26 & 223 & 20,8 \\
\hline Juillet. . . . . . . . & 0,1 & 230,4 & 25,2 & 15,1 & 254,1 & 21,9 & 12 & 276 & 23,3 \\
\hline Août. . . ........ & 3,6 & 216,6 & 23,9 & 26,7 & 190,6 & 22 & 33 & 246 & 22,8 \\
\hline Septembre. . ..... & 46,8 & 153,1 & 19,9 & 43,4 & 124,5 & 20,9 & 63 & 175 & 19,9 \\
\hline Octobre. . . ........ & 7,2 & 116,2 & 18 & 103,4 & 115,5 & 17,7 & 71 & 125 & 15 \\
\hline Novembre. . . ..... & 70,1 & $110\left(^{* *}\right)$ & 12,7 & 111,6 & $80\left(^{* *}\right)$ & 8,2 & 57 & $80\left({ }^{*}\right)$ & 10,2 \\
\hline Décembre. . . ..... & 10,8 & $100(* *)$ & 4,6 & 15,8 & $70\left({ }^{\star *}\right)$ & 7,6 & 62 & $70(* *)$ & 6,9 \\
\hline $\begin{array}{l}\text { Total/année, ou } \\
\text { moyenne de la } \\
\text { température. ..... }\end{array}$ & 221,7 & 1860 & 15,15 & 547,8 & 1584 & 14,89 & 524 & 1768 & 14,23 \\
\hline Déficit $(\mathrm{mm}) \ldots \ldots$ & \multicolumn{2}{|c|}{1640} & & \multicolumn{2}{|c|}{1036} & & \multicolumn{2}{|c|}{1244} & \\
\hline
\end{tabular}

\section{Bilan d'exploitation des usines de la Basse-Durance}

(fig. $1 d$ )

Les apports naturels en Durance ont été, de ce fait, assez pauvres et les déstockages de la réserve agricole du barrage de Serre-Ponçon ont joué pleinement leur rôle, en évitant à l'agriculture de subir une sécheresse catastrophique. Il en est résulté, pour les usines de Salon et Saint-Chamas, des apports très faibles, puisque durant une année complète elles n'ont turbiné qu'un volume total de 1430000000 de mètres cubes alors que pendant les neuf derniers mois de 1966 les apports avaient atteint 1900000000 de mètres cubes.

Le tableau 2 indique les apports des années 1966 et 1967 et nous y avons ajouté les chiffres théoriques d'une exploitation fictive qu'aurait eu l'usine de Saint-Chamas si elle avait fonctionné en 1961 , année dont l'hydraulicité fut très proche de la moyenne connue pour la Durance. C'est d'ailleurs l'année 1961 qui servit d'anmée d'exploilation type pour les essais sur le modèle réduit de l'étang de Berre; les volumes indiqués sont exprimés en millions de mètres cubes.

\section{Etude sur modèle réduit}

Le modèle, réalisé au Laboratoire de Chatou, représentait la totalité de l'étang de Berre, le canal de Caronte et le golfe de Fos. Les échelles principales de similitude étaient les suivantes:

- Iongueur en plan. . 1/750 distorsion : 7,5
- hauteur. . ...... $1 / 100$

- temps. . . ...... 1/75

- vitesse des courants. $1 / 10$

- débits. . ........ 1/750000

- masses spécifiques. . 1/1

- salinité. . ...... 1/1

Dans ces conditions, la période de la marée est réduite à $10 \mathrm{mn}$ sur le modèle.

Le modèle comportait un système de citernes, de tuyauteries et de pompes permettant la fabrication des eaux à différentes salinités.

Un générateur de marée pneumatique reproduisait en similitude la marée dans le golfe de Fos à l'aide d'un lecteur de came optique établie à partir des enregistrements in situ de la marée à Fort-deBouc.

Le modèle était équipé de six marégraphes à pointe vibrante, implantés sur le modèle, aux emplacements respectifs de ceux des appareils implantés in situ.

Les vitesses des courants de direction constante étaient mesurées au moyen de micromoulinets suffisamment petits pour ne pas introduire de perturbations, notamment dans le canal de Caronte.

Sur Ie modèle, la salinité $\left(^{*}\right)$ était déterminće par la mesure soit de la densité, soit de la conductivité électrique avec correction thermométrique pour les deux méthodes.

(*) Toutes les mesures de salinité indiquées dans le texte sont exprimées en \%, rapport entre la masse totale de sel dissous et la masse correspondante d'eau saumâtre, par exemple 38,5 grammes de sel dissous dans un kilogrammo d'eau de mer. Cette défnition a l'arantase d'être indépendante de la température. 
1 a/ Précipitations pluviométriques mensuelles.

Monthly rainfall.

$1 \boldsymbol{b} /$ Températures mensuelles moyennes.

Mean monthly temperature.

1 c/ Evaporations mensuelles. Monthly evaporation.

$1 \mathrm{~d} /$ Apports turbinés mensuels à l'usine de Saint-Chamas.

Monthly turbine flow volumes at Saint-Chamas power plant.

1 e/ Variations de la salinité des eaux superficielles près des anciennes salines de Berre.

Surface water salinity variation near the former Berre salt pans.
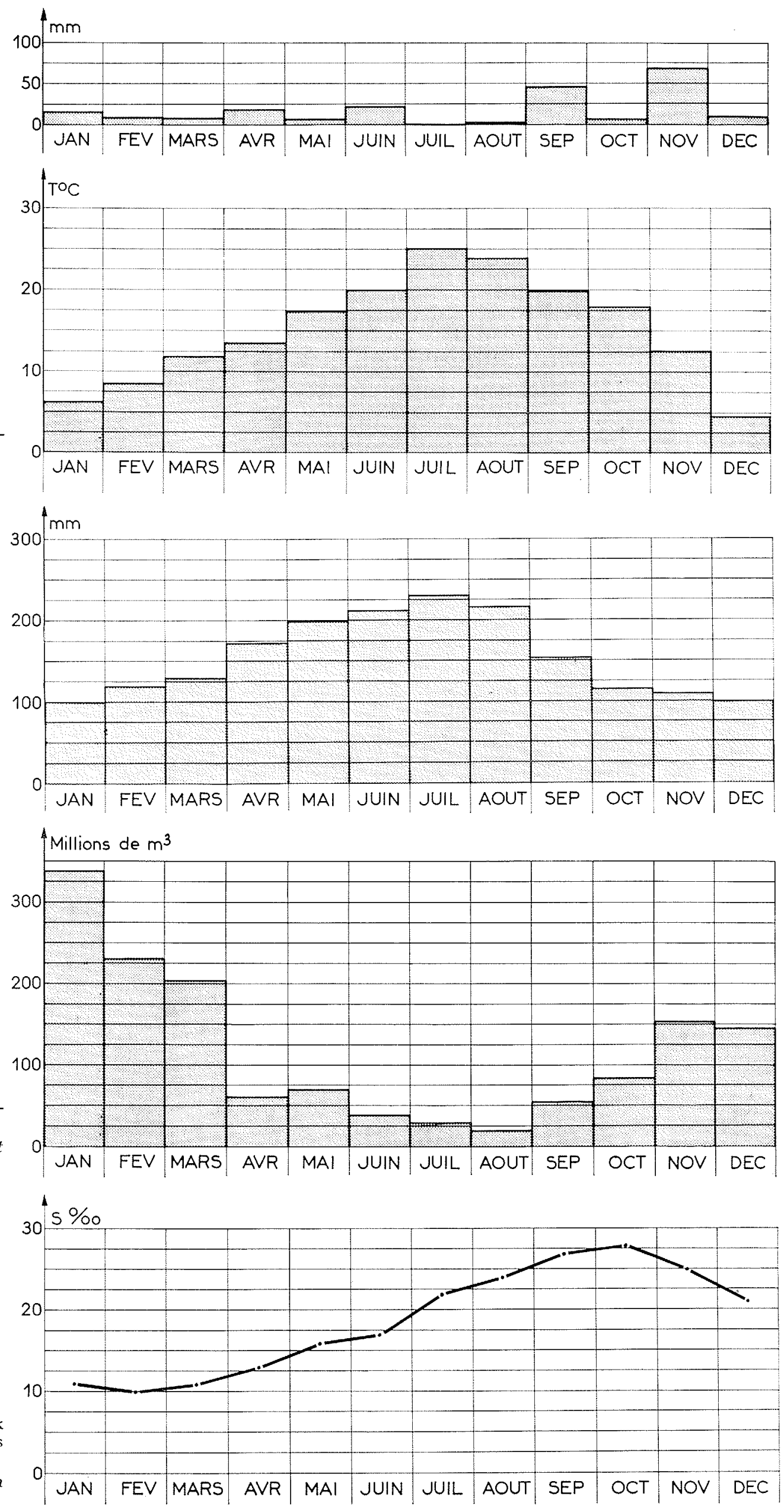


\begin{tabular}{|c|c|c|c|}
\hline \multicolumn{4}{|c|}{$\begin{array}{r}\text { Tableau } 2 \\
\text { Exploitation de l'usine de Saint-Chamas } \\
\text { (en millions de mètres cubes) }\end{array}$} \\
\hline ANNÉE & 1961 & 1966 & 1967 \\
\hline Janvier. . . . . . . . & 516 & 一 & 339 \\
\hline Février. . . . . . . & 442 & 一 & 231 \\
\hline Mars. . . . . . . . & 253 & $27,5\left(^{*}\right)$ & 203,9 \\
\hline Avril. . . . . . . & 198 & 250 & 62 \\
\hline Mai. . . . .......... & 243 & 265,8 & 69,6 \\
\hline Juin. . . . . . . . & 484 & 214,8 & 39,3 \\
\hline Juillet. . . . . . . . & 197 & 95,5 & 29,3 \\
\hline Août. . . . . . . . & 47 & 59,4 & 19,6 \\
\hline Septembre. . . .... & 33 & 11 & 53,4 \\
\hline Octobre. . . . . . . . & 191 & 247,4 & 84,8 \\
\hline Novembre $\ldots \ldots \ldots$ & 510 & 343.9 & 153,3 \\
\hline Décembre. . . . . . . & 457 & 359.9 & 144,8 \\
\hline Total. . . . . . . & 3573 & 1905 & 1430,5 \\
\hline $\begin{array}{l}{ }^{(*)} \text { Mise en service } \\
21 \text { mars } 1966 .\end{array}$ & e l'usir & de Saint-C & Chamas : \\
\hline
\end{tabular}

\section{Etalonnage du modèle}

En première approximation, un étalonnage en eau douce fut réalisé afin d'une part, d'adapter le générateur de marée à la reproduction correcte du phénomène à Fort-de-Bouc et, d'autre part, de déterminer les rugosités singulières à mettre en place sur le modèle et en particulier dans le canal de Caronte pour compenser l'effet de la distorsion des échelles de similitude.

L’étalonnage fut ensuite poursuivi en eau salée, avec reproduction des marées relevées pendant les campagnes de mesure in situ de mars 1961 et juillet $\mathbf{1 9 6 2}$.

A la suite d'une nouvelle campagne de mesures in situ en mars 1963, beaucoup plus complète que les précédentes, un complément d'étalonnage ful apporté au modèle.

Ces mesures étaient destinées à mieux connaître les répartitions verticales des vitesses et des salinités aux points caractéristiques suivants du canal de Caronte : passe de Port-de-Bouc, entrée du canal au droit de l'anse Aubran et passe de Jonquières.

Des mesures complémentaires de salinité eurent lieu dans l'étang et à l'intérieur du port de Lavéra.

Ces mesures ont été reproduites sur le modèle selon deux séries d'essais:

- pour la première série, la rugosité en place dans le canal était formée d'une couche de métal déployé placée au fond et d'un ensemble de tiges de laiton de $3 \mathrm{~mm}$ de diamètre constituant une rugosité dans la masse liquide;
- pour la seconde série, la rugosité était constituée seulement par une couche de métal déployé au fond du canal, les tiges étant supprimées.

Les deux séries d'étalonnage, avec el sans rugosité dans la masse donnaient des résultats salisfaisants et comparables en ce qui concerne la marée et les courants (fig. $2 a$ ). Par contre, les résultats des mesures de salinité ont été plutôt améliorés par la suppression de la rugosité dans la masse (fig. 2 b) et on pouvait considérer que le modèle était capable de reproduire avec une bonne précision les phénomènes étudiés.

\section{Conditions aux limites}

Si les conditions aux limites hydrauliques sont faciles à réaliser, il en est tout autrement de celles relatives à la salinité. En effet, lorsque l'usine de Saint-Chamas fonctionne, il est nécessaire d'évacuer à la limite maritime du modèle réduit un débit équivalent à celui de l'usine et cette évacuation ne pouvait s'effectuer qu'en eau de mer, ce qui aurait pour effet d'appauvrir le modèle en eau salée. Il faudrait donc reproduire exactement les échanges existants à la limite maritime du modèle, mais c'est précisément le phénomène que l'on n'aurait connu qu'après la mise en service de l'usine.

On a tourné la difficulté en partant de l'hypothèse que les surfaces isohalines dans le golfe de Fos n'étant pas verticales (ce mélange incomplet des eaux a été observé assez loin de l'embouchure du Rhône), on pourrait approcher d'assez près la condition idéale en faisant un soutirage en surface le long de la limite maritime du modèle mais avec un débit plus grand que celui de l'usine et en compensant par le fond cet excès d'aspiration par un apport d'eau de mer.

\section{Résultats des essais}

Seuls les phénomènes de convection sont correctement reproduits sur le modèle, soit par l'arrivée d'eau douce de l'étang à l'usine, soit par les courants de marée dans le canal de Caronte. Par contre, les phénomènes de mélange (en particulier le plus important est provoqué par le vent) ne peuvent être représentés sur le modèle que par un brassage mécanique artificiel dans l'hypothèse d'un mélange complet.

Une série d'essais préliminaires fut effectuée pour mettre au point d'une part, les méthodes de mesure el d'autre part, le maintien de la condition de salinité à la limite maritime du modèle.

\section{Essais de mise en service de l'usine (sans mélange)}

La salinité de l'eau du golfe de Fos étant réglée à $38,5 \%$ et celle de l'étang à $32 \%$, on réalisait en permanence la suite de deux marées relevées à Fort-de-Bouc le 18 juillet 1962 avec un débit d'usine constant à $200 \mathrm{~m}^{3} / \mathrm{s}$. Cet essai fut effectué deux 
La figure $3 b$ illustre l'évolution de la salinité en surface et en fond à Ia Pointe de Berre. On constate que l'étang se ressale beaucoup moins vite qu'il ne s'était dessalé et les lois de variation de la salinité peuvent s'écrire :

$$
35-\mathrm{S}_{\mathrm{fond}}=\mathrm{S}_{0} e^{-t / 30}
$$

avec $: \mathrm{S}_{0}=35-20=15 \%$

$$
35-\mathrm{S}_{\text {surfice }} \# \text { constante }
$$

En conclusion, ces essais préliminaires, qui n'avaient d'autre but que de dégrossir le problème, montrent d'une part, que la diffusion moléculaire sur le modèle a très peu d'effet et, d'autre part, que l'évolution de la salinité s'effectue soit en surface, usine en service, soit en fond, usine arrêtée. Enfin, on constate que l'étang perd plus facilement son sel qu'il ne le récupère en période de non-fonctionnement de l'usine.

Par ailleurs, et dans l'hypothèse de temps calme, le modèle montre une tendance très forte à la stratification des eaux de l'étang selon leurs densités.

\section{Essais de longue durée.}

Pour tenir compte de tous les phénomènes de convection (marée, variation de niveau moyen, exploitation réelle de l'usine de Saint-Chamas) on a reproduit sur le modèle le fonctionnement de l'usine et la marée enregistrée pendant l'année 1961.

Les salinités initiales au début de l'essai étaient :

- dans l'étang, 30,5\% pour le premier essai, $32 \%$ pour le second;

- dans le golfe de Fos, 38,5\% constant.

Au cours du premier essai, les mesures de salinité a trois ou quatre profondeurs, en divers points de l'étang, étaient effectuées par prélèvement environ toutes les demi-heures, d'un échantillon d'eau dont la salinité était déterminée ultérieurement par la méthode conductivimétrique.

Dans le deuxième essai, compte tenu des enseignements tirés du premier, on a effectué les mesures en trois points de l'étang, trois dans le canal de Caronte et un dans le golfe de Fos.

On relevait toutes les demi-heures en chacun de ces points la répartition verticale de la salinité, au moyen de sondes conductivimétriques à soutirage, en explorant la hauteur totale.

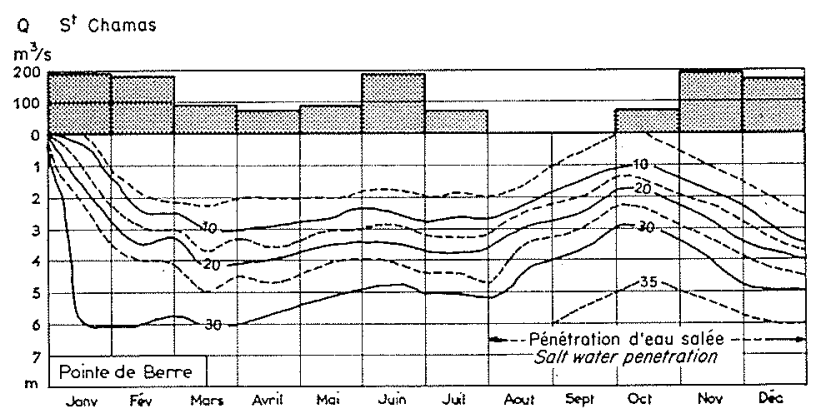

4/Evolution de la salinité a la Pointe de Berre durant l'année 1961 (Isohalines).

observed salinity at Pointe de Berre, 1961 (Isohalines).
Les conclusions des essais de longue durée ont été les suivantes:

- l'eau douce s'écoule à la surface d'un étang aux eaux se stratifiant par couches de plus en plus denses, donc plus salées en profondeur; on constate une pénétration lente d'eau salée à $35 \%$ et plus dans les zones profondes de la partie sud de l'étang par le canal de Caronte. La figure 4 montre l'évolution de la salinité à proximité de la Pointe de Berre. La zone de transition ou interface du coin salé se présente avec netteté sur le modèle avec une épaisseur quasi-constante de $3 \mathrm{~m}$. Cette stratification de l'étang a été réellement observée en périodes de temps calme (qui sont d'ailleurs peu fréquentes). Le facteur météorologique déterminant de cette région est en effet le vent, dont le brassage énergique des couches superficielles et profondes aboutil souvent à une salinité homogène du milieu;

- le vent ne pouvant être représenté en similitude sur le modèle, des essais ont été effectués dans l'hypothèse d'un mélange homogène des eaux par brassage mécanique. Dans ce cas, on peut évaluer les salinités finales d'équilibre de l'étang pour divers fonctionnements de l'usine de SaintChamas :

- pour un débit permanent de l'usine de $100 \mathrm{~m}^{3} / \mathrm{s}$, la salinité finale est d'environ de $3 \grave{a}$ $4 \%$ dans la zone nord de l'étang;

- pour un fonctionnement de l'usine à $200 \mathrm{~m}^{3} / \mathrm{s}$ durant six mois, suivi d'un arrêt complet de six mois, la salinité remonte lentement, mais sans dépasser 15 à $20 \%$ dans la zone sud de l'étang.

Ces résultats n'avaient pu être observés en 1966 , l'exploitation de l'usine de Saint-Chamas ayant été incomplète et assez différente de celle de 1961. En 1967, année exceptionnellement sèche et déficitaire, encore plus éloignée de l'année moyenne, il n'a pas été davantage possible de retrouver les résultats du modèle dans les différentes mesures in situ.

\section{Salinite des eaux de l'étang de Berre au cours de 1967}

On observe dès le printemps 1967 un ressalement spectaculaire de l'étang. La figure $1 e$ souligne la remontée rapide de la salinité à plus de $13 \%$ en avril, à près de $23 \%$ en juillet et $28 \%$ en octobre près des Salins-de-Berre. L'évolution des salinités dans les diverses zones de l'étang est illustrée par la figure 5 .

Même en fin d'année, où les apports dans le canal de la Basse-Durance augmentent à nouveau lentement, mais en restant très inférieurs aux valeurs mensuelles de l'année hydrologique moyenne, l'abaissement de la salinité relevé aux Salins-deBerre reste peu marqué. 
5/ Variations de la salinité des eaux superficielles en diverses zones de l'étang au cours de 1967.

Surface water salinity variation in the etang de Berre in $196 \%$.

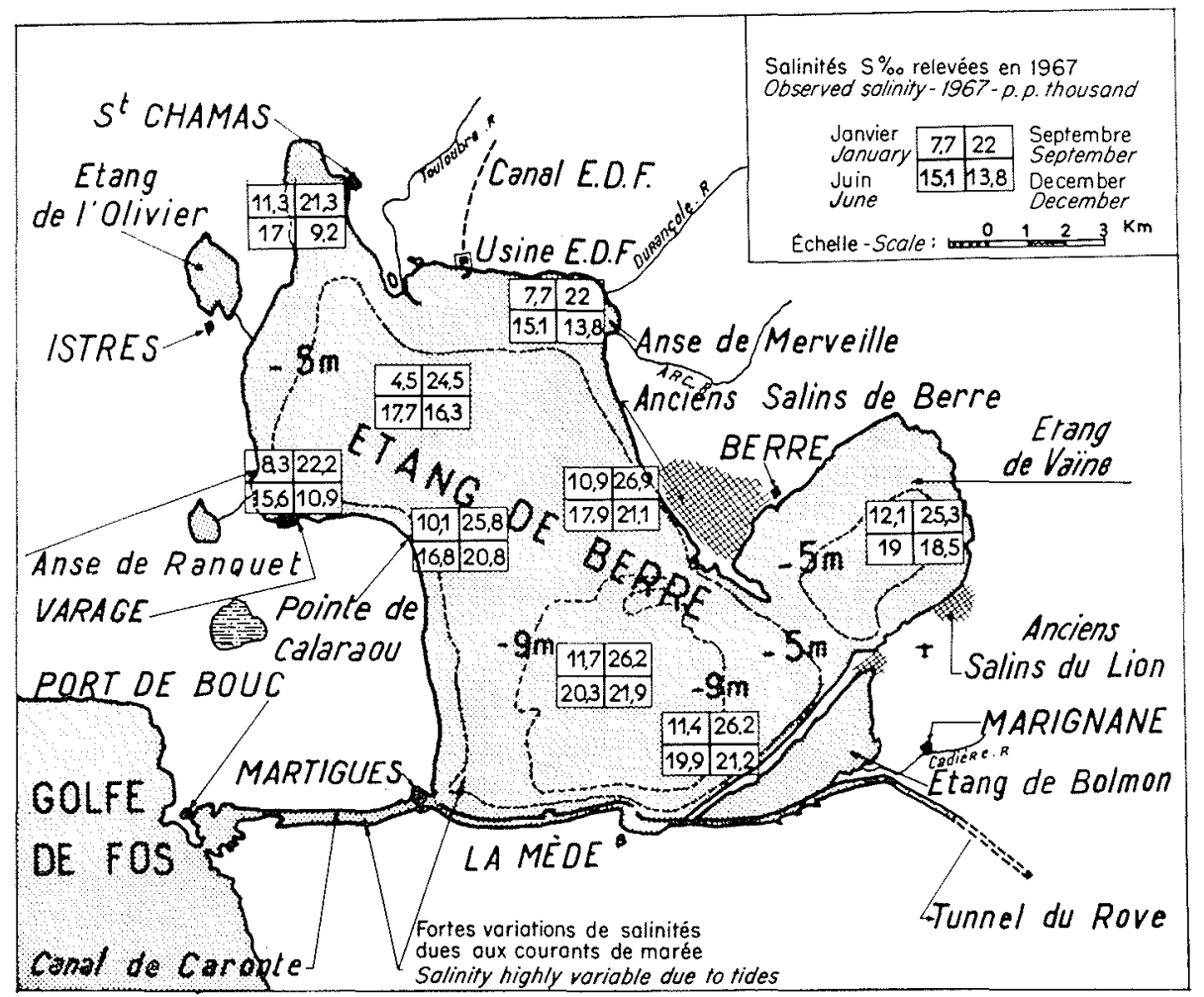

\section{Résultats \\ et "surprises" en matière de mesures de la salinité}

Si, dans l'ensemble, la salinité des eaux de l'étang décroît assez régulièrement de Martigues vers la zone de l'usine en période de fonctionnement de cette dernière et par temps calme, il n'est pas surprenant de constater un certain retard pour les variations de salinité des deux zones en «cul-desac » de la baie de Saint-Chamas et de l'étang de Vaïne.

Mais bien des surprises nous sont réservées quant aux résultats des relevés, en raison de la fantaisie des vents et d'autres facteurs que nous allons rapidement passer en revue:

\section{Vents, courants et seiches}

Parmi les vents, le mistral joue évidemment un rôle primordial à la fois par sa fréquence et par son intensité. Berre est un étang à fond pratiquement plat, de très faible profondeur (max. $10 \mathrm{~m}$ ) par rapport à ses dimensions (distance Martigues Port-de-Bouc $=9500 \mathrm{~m}$ ) et les vents forts agitent l'eau sur une bonne partie de leur épaisseur en bouleversant rapidement la stratification des eaux qui s'établit par temps calme. 1967 a été une année avec relativement moins de vent que les autres années et nous avons plusieurs fois pu constater une stratification marquée des eaux par couches de densité. C'est ainsi, par exemple, que le 13 février les couches superficielles dans la zone centre-sud avaient une salinité de $17,50 \%$ alors qu'à $-10 \mathrm{~m}$ celle-ci était de $31,20 \%$. Plusieurs jours après, après quelques fortes bourrasques, les salinités des eaux de surface et $\mathrm{du}$ fond étaient respectivement de 20,31 et 22,0\%. Quel chiffre donner alors pour une moyenne de salinité avec les multiples variations de ce genre? Les roses des vents fournies par les figures $6 a$ et $6 \mathrm{~b}$ respectivement pour les années 1966-1967, et pour la période 1960-1966, nous donnent une idée approximative des possibilités de bouleversements apportés par les vents à ce vaste plan d'eau. A ceux-ci s'ajoutent des mouvements provoqués alternativement ou simultanément par le phénomène de marée, des variations de niveau moyen et des oscillations propres du bassin, appelées « seiches».

En général, une dépression barométrique sur la région méditerranéenne s'accompagne d'un « coup de mistral $\gg$ dont les effets sur le plan d'eau masquent complètement le phénomène de marée :

- le niveau moyen baisse, la Méditerranée basculant vers les côtes d'Afrique, l'étang a tendance à se vider;

- le plan d'eau présente une inclinaison stable dont l'effet peut se faire sentir pendant plusieurs jours, la zone située au vent étant moins élevée que celle située sous le vent; la pente de la surface est fonction de la force et de la direction du vent; le vent souffle dans l'axe longitudinal de l'étang, ce qui tend à rendre son action plus efficace.

Le niveau s'élève fréquemment de plus d'un mètre en bordure de la digue du Jä̈ et l'eau de Berre entre en masse dans l'étang de Bolmon. Les bas quartiers de Martigues sont parfois inondés. 
- au creux de la dépression barométrique s'amorce une série d'oscillations libres du bassin, d'une heure de période environ et d'une dizaine de centimètres d'amplitude, en opposition de phase aux extrémités nord et sud de l'étang.

Par vent d'est ou de sud-est ( vent marin »), l'examen des marégraphes conduit aux constatations suivantes:

- élévation importante du niveau moyen, due aux basculements de la Méditerranée vers les côtes européennes;

- pente légèrement ascendante du plan d'eau dans le sens du vent. Le 21 septembre 1967, nous avons pu constater, sous l'effet de ce vent, une entrée d'eau importante de Berre dans le petit étang de l'Olivier dont les eaux peu salées (généralement 1 à $3 \%$ ) étaient littéralement « injectées 》 avec une eau à $18 \%$.

Des inondations se produisent parfois dans ces cas sur les quais de Saint-Chamas, d'autant plus que l'anse de Saint-Chamas, relativement étroite, a tendance à accroître l'amplitude des houles qui y pénètrent.

- apparition des seiches au moment du creux de la dépression barométrique. L’ingénieur-hydrographe Bourgoin (1966) en effectuant le calcul par la méthode de Sterneck à partir d'enregistrements, a Iocalisé la nodale entre la Pointe de Calaraou et l'extrémité nord de l'ancien salin de Berre. La période de la seiche était de $1 \mathrm{~h} 10 \mathrm{mn}$ environ, en bon accord avec celle observée sur les marégraphes.

Chevallier, dans son étude déjà fort complète de 1916, parle des courants qui ont lieu dans l'étang, mais il est impossible de dégager une loi en face de la complexité et des variations fréquentes des phénomènes : courants le long des rives, changement de direction et division des masses d'eau pour certains courants tels que celui venant le long de la côte est et dirigé par la Pointe de Berre vers la digue du Jaï... C'est ainsi que le vent venant du nord-est pousse les eaux superficielles de la zone centre-nord vers la côte de Varrage et au fond de l'anse du Ranquet d'où elles remontent le long de la côte ouest. Il en résulte que des filets, «calés» le long de cette côte, ont été plusieurs fois entrainés vers le nord, à contre-vent !

\section{Sources d'eau souterraines}

Mais les surprises les plus curieuses (et les plus déroutantes au début de nos mesures) sont dues à la présence de sources souterraines noyées. Chevallier en signale une dans la zone de Saint-Chamas sous le nom local bien connu de «Laouroun» et nous n'avons pas été étonnés de trouver, tout près de la petite digue de la pointe de Cadereau, des eaux presque douces $(3 \%)$.

Les salinités relativement faibles constatées sur les bords de l'anse de Ranquet sont probablement dues à des arrivées d'eau douce par résurgences noyées. Celles des eaux prélevées à la pointe de Calaraou s'expliquent soit par l'arrivée d'eau dessalée venant de l'anse de Ranquet, soit par la présence locale de résurgences noyées.
Mais quel ne fut pas notre étonnement de constater, en plein centre de l'étang de Vaïne, des eaux à $5,2 \%$ en surface et $3,9 \%$ en profondeur (à - 4,20 m), alors que la salinité du reste de l'étang était partout voisine de $19 \%$ (en juin 1967). Nous avions, sans le savoir, fait notre prélèvement au voisinage immédiat de l'une de ces sources débouchant dans les fonds de l'étang.

La présence de ces résurgences n'a rien d'extraordinaire; à l'époque préflandrienne, la Méditerranée était d'environ $40 \mathrm{~m}$ plus basse que le niveau actuel des mers (Corroy, 1958) et de nombreux "boyaux » karstiques de cette époque (canaux creusés dans les formations calcaires) sont noyés, mais servent toujours à certains mouvements d'eau souterraine.

Facteurs divers

Dans l'interprétation des mesures de salinités, i] y a lieu de tenir compte des arrivées d'eau douce: essentiellement Touloubre, Durançole (et quelques autres petites résurgences voisines de l'anse de Merveille), Arc. La Touloubre est en partie canalisée vers les poudreries de Saint-Chamas et cette déviation adoucit les eaux du fond de la baie. Enfin, avant la fermeture des Salines (Berre et Lion), les prélèvements faits à leur voisinage devaient être opérés en s'assurant qu'aucune saumure n'avait été rejetée dans l'étang: en effet, après évaporation des eaux dans les partennements et dépôt du chlorure de sodium, les salines rejetaient de temps en temps les eaux mères résiduelles encore très riches en sels, surtout de magnésium.

\section{Echanges d'eau entre l'étang et le golfe de Fos}

Fèbre et Mars (1965, référence note précédente) ont mis en relief l'importance des volumes d'ean échangés entre l'étang et le golfe de Fos par suite du jeu des marées. Pour la période voisine de 1964 , ces auteurs estiment à environ quatre milliards de mètres cubes le volume transitant chaque année par Martigues dans le sens mer-étang et à 4,25 milliards en sens inverse. Ces valeurs sont fortement augmentées de nos jours avec les apports importants de Saint-Chamas. En effet, l'interface séparant les eaux dessalées sortantes des eaux plus salées qui entrent par courant de densité, est d'autant moins inclinée que les différences de salinité (donc de densité) entre les deux masses d'eau sont fortes. Plus l'apport d'eau douce par Saint-Chamas est élevé, plus l'interface s'allonge en diminuant de pente et permet paradoxalement une pénétration plus importante d'eau de mer venant du golfe de Fos. A noter de plus que l'interface se déplace alternativement avec les courants de marée. La figure 7 schématise ce phénomène. La figure 8 indique schématiquement le bilan des apports d'eaux douces et salées dans l'étang et les pertes par évaporation. 


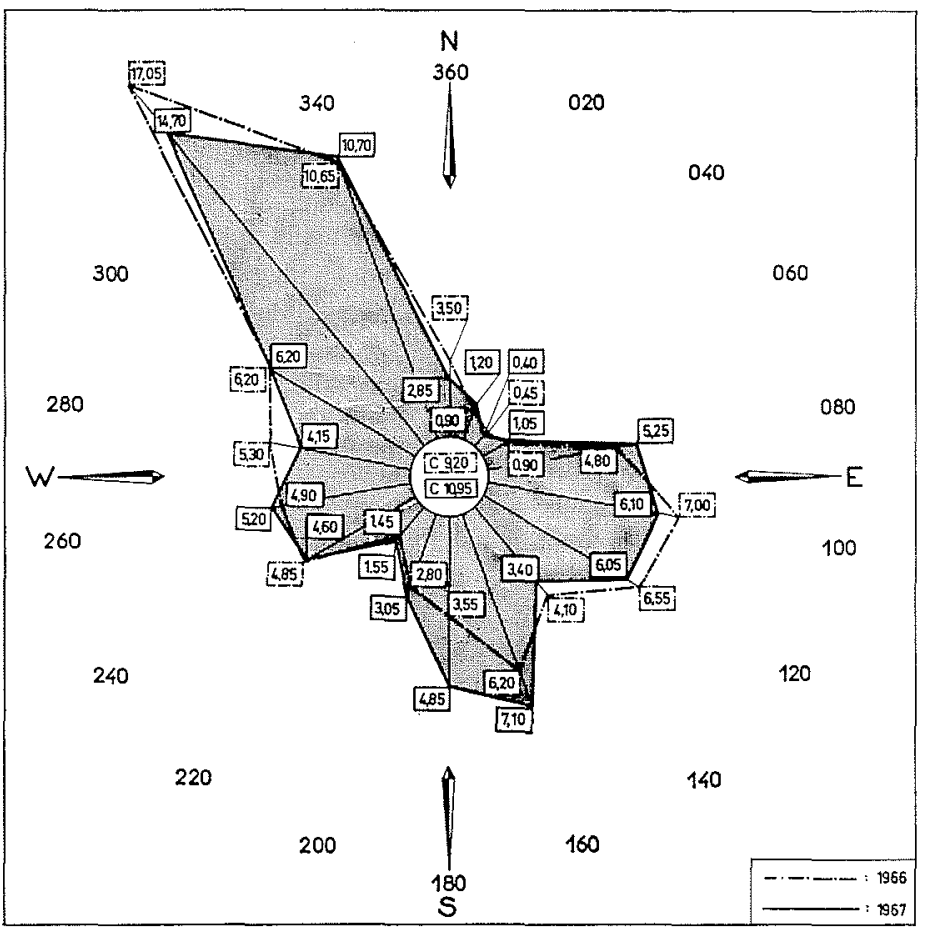

6 a/ Rose des vents 1966 et 1967 (fréquences en $\%$ ). Wind frequency (percent) diagram for 1966 and 1967.

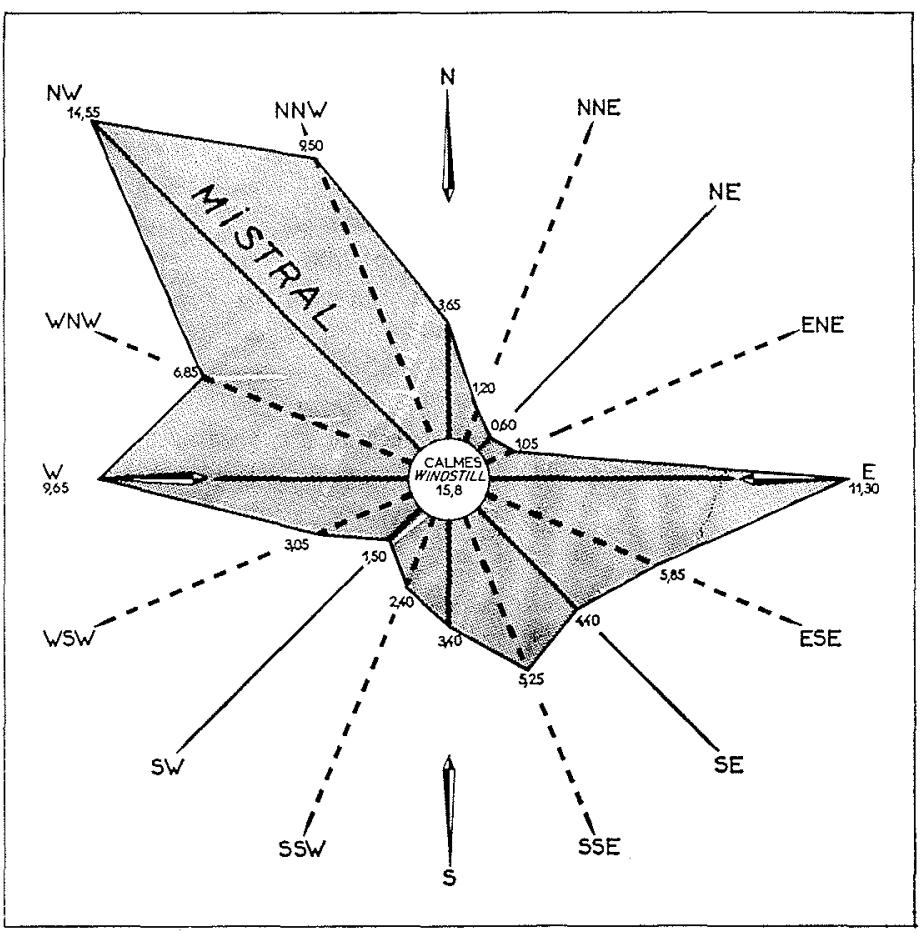

6 b/ Rose des vents. Période 1960-1966 (fréquences en \%). Wind frequencly (percent) diagram for 1960-1966.

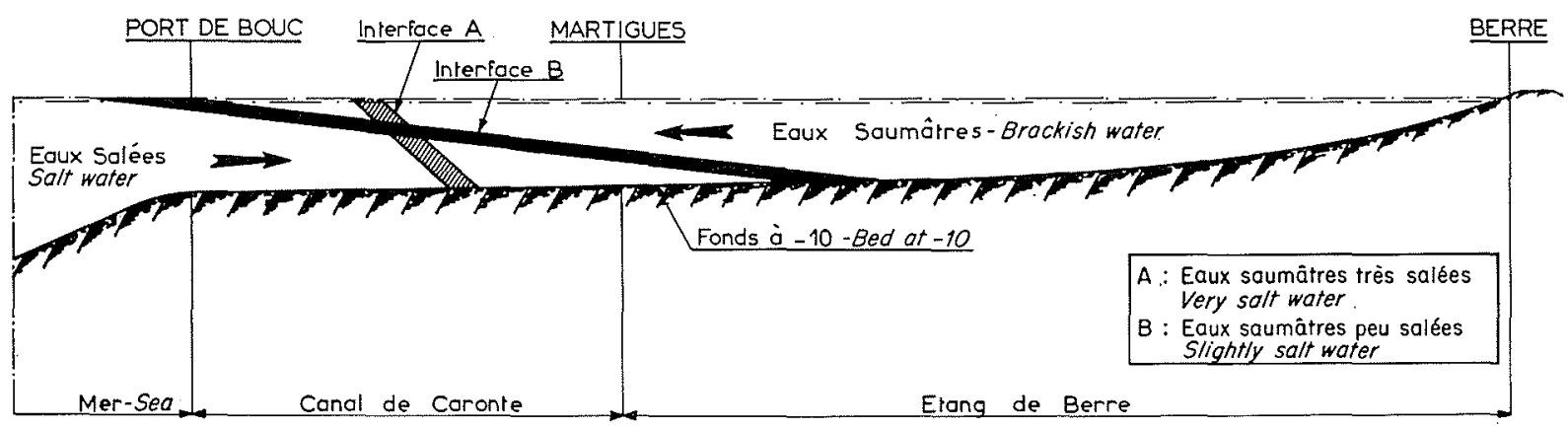

7/ Modification schématique de l'interface du coin salé selon le gradient de salinité.

Diagrammatic salt wedge interface variation with salinity gradient.

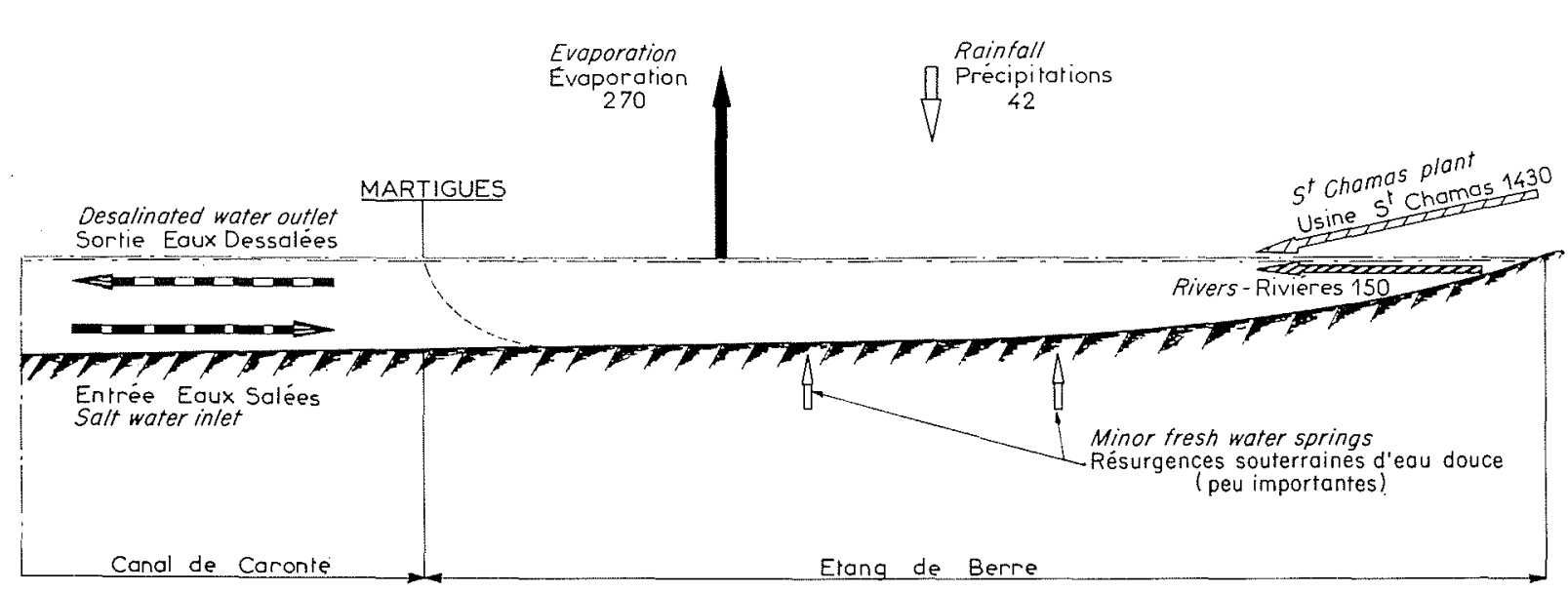

8/ Bilan schématique des apports d'eaux douces et salées, et des pertes par évaporation pour l'ensemble de l'étang de Berre, pour l'année 1967 (en millions de mètres cubes).

Overall fresh and salt water inflows and evaporation losses for the étang de Berre in 1967 . (Figs. $\times 10^{\circ}$ cubic metres). 
Les résultats obtenus sur modèle ne peuvent donc nous donner qu'une indication approximative, d'autant plus que le phénomène de rétention des sels par les fonds (vases salées) joue légèrement en faveur d'un retard au dessalement de l'étang.

Signalons, enfin, que le canal du Rove (Gaver et Timon David, 1937) dont le percement avait eu pour conséquence d'augmenter la salinité de l'étang de Berre, est effondré depuis plusieurs années dans sa partie centrale et, de ce fait, n'a plus aucune influence sur les eaux de l'étang.

\section{Observations sur le plan biologique (poissons) (*)}

Dans la moitié sud de l'étang, relativement profonde et à salinité toujours restée élevée (Martigues, zone centre sud, digue du Jaï) les espèces ichtyologiques n'ont pratiquement pas changé, à part quelques rares exceptions telles que la disparition déjà signalée pour les hippocampes [1] dont quelques exemplaires sont cependant encore capturés dans le canal de Caronte.

Dans la moitié nord, soumise au cours de 1966 et tout début 1967 à un fort dessalement, puis à un ressalement important au cours de l'été et de l'automne 1967, nous pourons résumer nos observations comme suit :

\section{Espèces euryhalines}

De par leur indifférence vis-à-vis de la salinité du milieu, les poissons suivants sont toujours présents : anguille [2], mulets [3 à 5], loup [6], athérine de Boyer [7], alose [8] qui passe dans l'étang aux moments de sa migration vers ses frayères en eaux douces, flet [9]. Ces espèces existent aussi dans le petil étang peu salé de l'Olivier (salinité 1 à $3 \%$ ).

\section{Espèces à affinités marines dominantes, mais supportant, pour un certain temps du moins, une eau assez fortement dessalée (jusqu'à environ $10 \%$ )}

Parmi ces poissons voyageant entre la mer et l'étang, signalons d'une part, plusieurs espèces de la famille des Sparidés : saupe [10], daurade [11], sargues ou sars $[12$ et 13], marbré [14] et, d'autre part, la sole commune [15] et l'orphie [16]. Ces divers poissons qui ne pénètrent que de facon très irrégulière dans les eaux dessalées, avaient diminué en nombre dans la zone nord de l'étang en automne 1966 et ceci avant l'époque où la majorité d'entre eux ont l'habitude de retourner à la mer; le ressalement important de cette zone a permis leur réapparition après mars 1967 .

$\left(^{\star}\right)$ Les noms seientifiques des poissons cités ont été regroupés à la fin de cette note et nous avons donné, pour chacun d'eux, le synonyme provençal le plus courant.

\section{Espèces sédentaires exístant aussi en mer, mais pouvant effectuer leur cycle biologique dans les eaux saumâtres}

Dans ce groupe, citons plusieurs Gobiidés, Syngnathidés et Labridés plus ou moins résistants au dessalement :

- parmi les Gobiidés, quatre espèces sont fréquentes dans l'étang: le moulet d'assez grande taille [17] (jusqu'à $20 \mathrm{~cm}$ ), le gobie noir [18] atteignant $15 \mathrm{~cm}$ et deux mougnettes $[19 \mathrm{et}$ 20] qu'il est facile de confondre et qui ne dépassent pas $6 \mathrm{~cm}$. Seules les deux dernières supportent des dessalements importants (moins de $10 \%$ ) et elles sont toujours restées assez abondantes. Les deux premières, après avoir diminué fin 1966, ont reparu normalement au cours de l'été 1967. En plus de ces espèces, signalons la mougne claire [21] qui, plus sensible que les autres espèces au dessalement des eaux, était capturée sur fonds sablonneux dans le nord (en particulier anse de Merveille) où elle n'a plus été rencontrée depuis l'automne 1966;

- parmi les Syngnathidés, le siphonostome [22] et le grand syngnathe [23] sont les plus sensibles au dessalement, quelques sujets seulement ont reparu dans la zone nord au cours de l'été 1967. Par contre, le petit syngnathe [24] est resté abondant dans tous les herbiers auxquels le ressalement a donné une nouvelle vigueur;

- parmi les Labridés, la canadelle [25] était autrefois particulièrement abondante dans tout l'étang et elle s'était raréfiée fin 1966. L'été 1967 a permis sa réapparition en assez grand nombre, mais, avec la marche normale de l'usine, elle va à nouveau se raréfier dans la zone nord;

- enfin il y a lieu de signaler, dans ce groupe, la bavarelle [26] qui, elle aussi, a connu une raréfaction avec le dessalement des eaux et un nouveau regain d'abondance dans la zone nord au cours de l'été et l'automne 1967.

\section{Espèces à affinités marines dominantes, relativement sténohalines, mais pouvant faire des incursions dans les eaux saumâtres encore très salées}

Il est évident que ce groupe de poissons, que l'on ne rencontre guère dans les eaux de salinité inférieure à $25 \%$, avait totalement disparu fin 1966 dans la moitié nord de l'étang, où l'été 1967 à permis la réapparition de quelques sujets : rouget [27], melets [28 et 29], sardine [30], chinchard [31], muge à grosse lève [32]. Ce dernier, beaucoup plus rare que les trois autres espèces de muges déjà signalés, atteint des tailles voisines de $1 \mathrm{~m}$. 


\section{Liste des noms scientifiques des poissons cités}

[1] Hippocampus guttulatus Cuv. : Chivaou marin (Syngnathidé).

[2] Anguilla anguilla I : Anguillidé, mne seule espèce.

[3] Mugil cephalus L. : Muge cabot ou Testu

[4] Liza aurata (Risso) : Muge doré ou Gaouto rousso

[5] Liza capito (Cuv.) : Muge ramada on Mugrilidés. Pointu

[6] Morone Labrax L. : aussi dénommé Bar ou Loubas (Serranide).

[7] Atherina boyeri Risso : Joël ou Cabassoun (Athérinidé synonyme d'A.mochon C.).

[8] Alosa ficta rhodanensis (Roule) : Alauso ou Alatcho (Clupeidé).

[9] Flesus flesus L. : Variété méditerranéenne passer L. : larbe (Pleuronectidé).

[10] Boops salpa L. : Saoupa.

[11] Chrysophrys aurata L. : Aourado.

[12] Diplodus annularis L. : Sparaillon ou Pataclet.

[13] Diplodus sargus L. : Sargue de Rondelet.

[14] Pagellus momyrus C.V. : Mormo (plus rare que les sars).

[15] Solea vulgaris Quensel (Soleide) : Lingua.

[16] Belone belone L. (Scombrésocidé) : Grande aiguille on Aguio.

[17] Gobias ophiocephalas Pallas : Gobie lote ou Gobi.

[18] G. niger L. : Gobie jozo, Gobie à haute dorsale ou Gobi négré.

[19 et 20] G. microps Kröyer et G. marmoratus Risso.

[21] G. minutus Pallas : Petit sobie on Gobje buhotte.

[22] Syngnathas typhle L.

[23] S. acus I.

[24] S. abaster Risso
[25] Symphodus griseus (Gmelin) : Crénilabre (espèce aux couleurs particulièrement variées).

[26] Blennius pavo Risso : Blennie paon ou Biboué (Blenniidé).

[27] Mullus barbalus L. : Rouget barbet ou Testo plato (Mullidé)

[28] Clupea sprattus L. : Sprat (Clupeidé).

[29] Engraulis encrasicholus (L.) : Anchois (Engraulidé).

[30] Sardina pilchardus Walbaum : (Clupeidé).

[31] Trachurus trachurus L. : Saurel (Carangidé).

[32] Crenimugil chelo (Cuvier) : limpu ou œil négré (Mu. gilidé).

Bibliographie chronologique

Cette bibliographie ne reprend pas les références déjà données dans notre étude précédente de 1967.

Chevalder (A.). - L'Etang de Berre. Annales Inst. Océan., 7, $\mathrm{n}^{\circ} 4(1916), 91 \mathrm{p}$.

Gaver (F, Van) et Tmox David (J.) - Etude sur la faune du canal de Marseille at Rhóne entre l'Estaque et Portde-Bouc. Ann. Mus. Nat. Marseille, 28, no 2 (1937), 24 p.

Conhoy (G.), Gouvennet (C.), Chouteau (J.), Sivinine (A.), Griet (R) et Picalin (J.). - Les résurgences sous-marines de la région de Cassis-Monaco. Bull. Inst. Océan., $\mathrm{n}^{\circ} 1131$ (1958), $35 \mathrm{p}$

Boungon (J.). - Etang de Berre. Oseillation du plan d'eau et variations du nivea moyen. Cahier Océan. 18 (1966), $122 \mathrm{p}$.

Blanc (F.), Corst (B.), Minas (H.J.) et Szenielda (K. H.) Distribution et corrélations des principaux facteurs hydrobiologiques dans un milieu de forte production organicue (étang de Berre). Marine Biology. Intern Journ. Life Oceans and Coastalwaters, 1, no 1 (1967), pp. 43-55.

Kiendr (A.) et Longuemane (R.), - Evolution de l'étang de Berre en fonction des grands travaux d'equipement de l'Electricité de France sur la Durance. La Houlle Blanche, n ${ }^{\circ} 5(1967)$, pp. 507-513.

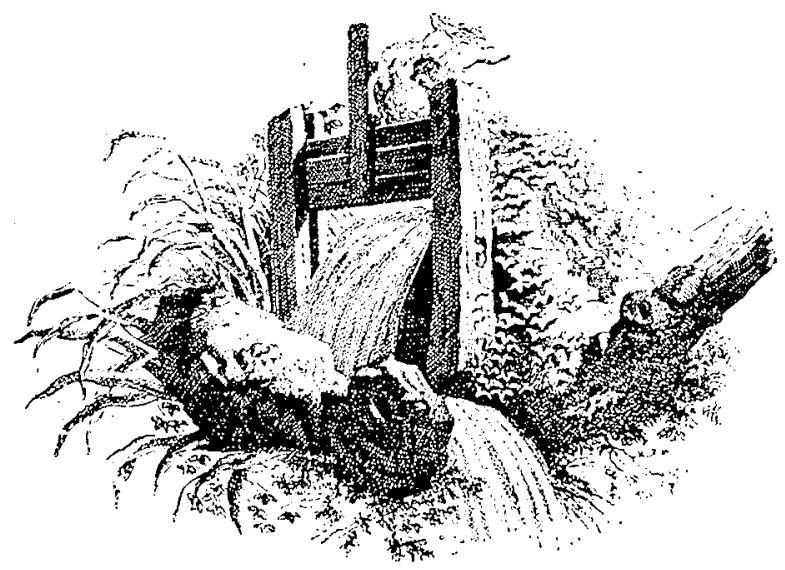

Gravure du XVIIIc siècle 